\title{
Bi-dimensional semantic scales: the embodied maps of meanings*
}

\section{Escala semántica bidimensional: La corporeidad de los mapas de significados}

Recibido: junio 1 de 2012 | Revisado: agosto 1 de 2012 | Aceptado: agosto 20 de 2012

\author{
PETAR MILIN ** \\ SUNČICA ZDRAVKOVIĆ **** \\ University of Novi Sad, Serbia \\ University of Belgrade, Serbia
}

\section{Doi:10.11144/Javeriana.UPSY12-5.bdss}

Para citar este artículo: Milin, P., \& Zdravković, S. (2013). Bi-dimensional semantic scales: the embodied maps of meanings. Universitas Psychologica, 12(5), 1543-1558. Doi:10.11144/Javeriana. UPSY12-5.bdss

Acknowledgements: This research was supported by the Ministry of Education and Science, Grants No. 179033, 179006, and III47020. The authors would also like to thank Stevan Radojević, who developed the application for a computer web-based experiment, to Fernando Marmolejo Ramos, the editor of the special issue, to Robyn Groves for proofreading this manuscript, and to two anonymous reviewers for valuable suggestions.

** Department of Psychology, University of Novi Sad, Serbia. Laboratory for Experimental Psychology, University of Belgrade, Serbia. E-mail: petar. milin@uni-tuebingen.de. Researcher ID: K-60572013.

**** Department of Psychology, University of Novi Sad, Serbia. Laboratory for Experimental Psychology, University of Belgrade, Serbia: E-mail: szdravko@f. bg.ac.rs. Researcher ID: K-7183-2013.

\begin{abstract}
A B S T R A C T
Osgood developed the semantic differential to bridge the phenomena from semantics and perception, and we applied its modified version to investigate current issues in cognitive science. We used two-dimensional rather than one-dimensional space to position nominal word items, and subjected data to multidimensional scaling (MDS). In Experiment 1 (paper-and-pencil) participants judged concrete and abstract nouns on seven bipolar semantic differential scales in three perceptual modalities: visual, auditory and touch. Six months later, in Experiment 2 (computer-assisted), the same participants mapped the same ten nouns on a balanced subset of two-dimensional planes. Our findings support the hypothesis that semantic space is physically constrained. MDS over one-dimensional ratings from Experiment 1 resulted in a particular two-dimensional solution. This two-dimensional combination was very similar to one of the raw two-dimensional maps from Experiment 2. We then concluded that this particular raw two-dimensional map is highly informative, as it captures almost all differences between word items in the given set of perceptual opposites. Its informativeness proved to be robust to experimental administration (paper-and-pencil vs. computerassisted) and scale-orientations (horizontal vs. vertical). Recent theories, such as Barsalou's perceptual theory of knowledge, capture the tradition of conceptualizing all knowledge as inherently perceptual. Our results strongly support these theories.

Key words author

Semantic space, semantic differential, multimodal perception, multidimensional scaling.

Key words plus

Perceptual Theory of Knowledge, Cognitive Science, Language.
\end{abstract}

\section{RESUMEN}

Osgood desarrolló el diferencial semántico para investigar los fenómenos desde la semántica y la percepción, y aplicamos su versión modificada para investigar temas actuales en ciencia cognitiva. Utilizamos dos dimensiones en lugar de un espacio unidimensional para posicionar palabras nominales y datos sujetos a escalamiento multidimensional (MDS). En el experimento 1 (papel y lápiz) participantes consideraron sustantivos concretos y abstractos en siete escalas de diferencial semántico bipolar en tres modalidades perceptuales: visual, auditivo y táctil. Seis meses más tarde, en el Experimento 2 (asistida por ordenador), los mismos participantes asignaron los mismos diez sustantivos en un subconjunto equilibrado de planos bidimensionales. Nuestros resultados apoyan la hipótesis de que el espacio semántico está limitado físicamente. Las calificaciones unidimensionales sobre MDS del Experimento 1 dieron como resultado una solución de dos dimensiones 
particular. Esta combinación de dos dimensiones fue muy similar a uno de los mapas de dos dimensiones en bruto del Experimento 2. Se concluyó que este mapa particular de dos dimensiones es altamente informativo, ya que captura casi todas las diferencias entre las palabras en el conjunto dado de los opuestos perceptuales. Esta información demostró ser sólida a la administración experimental (papel y lápiz versus asistida por computador) y las orientaciones de la escala (horizontal y vertical). Teorías recientes, como la teoría del conocimiento perceptual de Barsalou, captura la tradición de la conceptualización del conocimiento como inherentemente perceptual. Nuestros resultados apoyan firmemente estas teorías.

Palabras clave autores

Espacio semántico, diferencial semántico, percepción multimodal, escalamiento multidimensional

Palabras clave descriptores

Teoría perceptual de conocimiento, ciencia cognitiva.

\section{Introduction}

Recent years have witnessed a significant retreat from the idea that our cognitive functions are represented in a strictly symbolic mode. This rule of symbolic, amodal representations was a response to a previously dominant view that behavior is an optimal level of explanation in psychology. The strong need at that time, to establish the importance of higher cognitive functions, had the consequence that the mind as an emerging function of the brain, was forgotten. However, authors closer to perception always insisted on the importance of material body. Marr (1982) proposed that complete understanding of a function is possible only when we understand its implementation into a particular physical system. Along the same lines, action is considered to be a cognitive function, not a mere outcome of other, hierarchically higher, functions. In fact, it was proposed that the same computational processes underlie perception and higher forms of cognition, such as social cognition (Wolpert, Ghahramani, \& Flanagan, 2001). Therefore cognition is placed between the world and the body; in the center of information about the world, which is formatted through our senses, and our bodily motor interaction with that world.

In the field of cognitive psychology, grounded cognition represents a sharp theoretical turn in the direction that we have previously described. According to grounded cognition all cognitive processes are fundamentally dependent on bodily states (Barsalou, 2008; Gibbs, 2006). Above all, our phenomenology is shaped by these "embodied experiences". Our conscious cognition such as perception, thinking or language, all have the same embodied or sensorimotor grounding (Barsalou, 1999). Furthermore, related theories propose that even abstract concepts have their source in situated knowledge (among others, see Barsalou, 2005; Barsalou, 2009; Barsalou \& Wiemer-Hastings, 2005; Dove, 2011; Gibbs, 2006; Gleitman et al., 2005; Johnson \& Lakoff, 2002; Kousta, Vigliocco et al., 2011; Lakoff \& Johnson, 1999; Vigliocco et al., 2004; Wiemer-Hastings \& Xu, 2005).

It is interesting that this new approach has not been challenged with vast empirical data from the previous era. On the contrary, Barsalou (2008) criticized this lack of empirical support, and even attempts to test the previously dominant view of amodal representations. Again, in the field of perception, an attempt was made to relate cognition and body, or more precisely to establish the format of (abstract) concepts in terms of (perceptual) experience.

Years before, Osgood initiated a measurement of connotation of the concepts in an objective way, by using a technique that is a combination of scaling and association (Osgood \& Luria, 1954). Osgood (1952), and Osgood, Suci and Tannenbaum (1957) began a line of research in which the meaning of words - their semantics - was captured by a list of bi-polar scales. A large number of scales (50) was initially tested with two different factor analyses both converging to the same 3 factor solution (Osgood \& Suci, 1955). These scales heavily relied on experience, either of perceptual or abstract nature (e.g., high - low, kind - cruel, valuable - worthless etc.). Their results drew three important implications for semantic space modeling: (1) gradient nature of meaning is limited "by the sensory nervous system", while (2) many of the "experiential continua" are equivalent given "highly inter-correlated clusters" of "descriptive scales", thus, allowing for (3) "a limited number of such continua" defining the full-array of semantics (Osgood, 1952, p. 226227). 
Barsalou, in his perceptual theory of knowledge, expressed similar ideas stating that words are associated with concepts "to produce a semantic field that mirrors the underlying conceptual field" (Barsalou, 1999, p. 592; also in: Barsalou, 1991; 1992; 1993). According to the author's view, even abstract concepts and associated words are settled in the semantic field, which is constrained by a sensory input. However, while Barsalou (1999) may have found many theoretical arches between his theory of knowledge and traditional philosophical systems, from Aristotle and Epicurus to Russel and Price, he never related his work to the research of Osgood and his collaborators.

Barsalou's view of language concurs with more refined, modern approaches to word meanings. A good example is the rather detailed discussion of human acquisition of "easy" (concrete) words and "hard" (abstract) words by Gleitman and her collaborators (Gleitman et al, 2005). These authors have been focused on linguistic structures, "crucial in narrowing the way that the extralinguistic world itself is to be relevantly parsed", where a language learner must make "inductive inferences of great complexity and subtlety using, among other cues, the evidence of the senses" (Gleitman et al., 2005, p. 56). Importantly, the authors claim that mappings between the language units and the world are essential for learning both "easy" and "hard" words: while concrete words are first to be learned by direct word-to-world mappings, abstract words require structure-to-world mappings, which make use of more complex syntagmatic information.

Vigliocco et al. (2004) went even further by hypothesizing a unitary semantic space of word meanings. For those authors, a word meaning is grounded in feature-based conceptual representation, while words - lexical representations - themselves serve as an interface between (meaningful) conceptual representations and (structural) linguistic information, syntactic or phonological. Again, conceptual feature-based representations must have sensorimotor grounding, at least for concrete concepts.

In pursuing the same line of research and reasoning, Kousta, Vigliocco et al. (2011) showed that emotional valence and linguistic information are essential for abstract concepts. When discussing the findings of Gleitman and her collaborators (2005), the same authors concluded that abstract words that denote emotion-related states must also be "easy", allowing for word-to-world mappings (Kousta, Vigliocco et al., 2011; also consult Vigliocco, Meteyard, Andrews, \& Kousta, 2009).

From the previous brief presentation, we can conclude that many empirically driven frameworks of research in word semantics, such as those of Vigliocco et al. (2004) or Gleitman et al. (2005), harmonize with Barsalou's general theory of knowledge. Our present goal is to bring attention back to Osgood's methodology, which might provide yet another evidence for the same broad claim: there must be some grounding in a word's meaning.

\section{Theoretical issues: Embodied meaning}

Our theoretical understanding of cognition is deeply influenced by the ideas within grounded cognition framework. The present study is therefore an attempt to extend these ideas, and to give perceptual experiences a central role in shaping the conceptual space. However, it is important to clarify that, here, we understand conceptual space as a semantic memory for abstract concepts, constituting our thoughts, and that we leave propositionality beyond the current scope.

Since a conceptual representation of the world is not static but changes in time, driven by the incoming sequence of multimodal perceptual experiences, we believe that there must be a pervasive interaction among perception and higher cognitive functions such as language (more about the multimodal perception in: Ghazanfar \& Schroeder, 2006; Shimojo \& Shams, 2001; also, for the top-down effects of language on perception see: Huettig \& Altmann, 2011; Spivey, Tyler, Eberhard, \& Tanenhaus, 2001; Yee, Ahmed, \& Thompson-Schill, 2012; Lupyan, G. 2012). The dynamic conceptual space created from perceptual input is not dominated by the data from a single sense. It benefits from all available sense data. Therefore, data from the senses such as vestibular would not significantly influence the conceptual space, while those coming from the 
visual, auditory, tactile or chemical senses, ought to be determining. Naturally, not all of them would contribute equally to each object. For example, conceptual representation of a favorite food might be more influenced by the chemical rather than the auditory sense.

Again, there is a question of how abstract concepts and words are actually embodied. In many respects, this became one of the central issues of the grounded cognition framework and responds to this question range substantially. One approach to the abovementioned question follows Dual Coding Theory - DCT (see Paivio, 1986; and also Sadoski \& Paivio, 2004), proposing the "dis-embodiment" of a natural language, in the sense that language is an embodied symbolic system, but whose embodiment is arbitrary in relation to its semantics (see Dove, 2009; Dove, 2011). Barsalou, Santos, Simmons, \& Wilson (2008) proposed the Language and Situated Simulations (LASS) view on the same issue, which is related to the DCT, but is more inclined towards original grounded cognition claims. The authors see abstract concepts as situated, where events and introspections are taking most of their grounding (for similar discussions see also Barsalou, 2005; Barsalou, 2008; Barsalou, 2009).

A more empirically oriented view on the matters of abstract conceptualizations and their groundings is given by Wiemer-Hastings and her associates (Wiemer-Hastings \& Xu, 2003; Wiemer-Hastings $\& X u, 2005$; Barsalou \& Wiemer-Hastings, 2005). In a series of studies the authors showed that abstract concepts have greater variability. However, they show fewer intrinsic features than concrete concepts, hence, more dependency on item-external events and entities. This leads to their temporal and relational nature. These findings as described resonate with the Gleitman et al. (2005) structureto-world mappings for "hard", more complex words, or with the view of Vigliocco and her collaborators (2004) that lexical representations might serve as an interface for conceptual representations, for abstract ones in particular.

Finally, there is yet another grounded cognition stream whose central claim is that abstract concepts' embodiment is in metaphors, i.e., that metaphors make the semantics of abstract concepts more variable and flexible or "augmented" (Lakoff \& Johnson, 1980; Lakoff \& Johnson, 1999; Gibbs, 1994; Gibbs, 2006; with empirical support from Boroditsky \& Ramscar, 2002). This claim does not contradict the findings of Wiemer-Hastings and collaborators (Wiemer-Hastings \& Xu, 2005), and can be aligned with situated conceptualizations (Barsalou, 2005; Barsalou, 2009), but is less favorable under the dis-embodied view due to its developmental implausibility and to its direct alignment of linguistic metaphors and conceptual structures (for details consult Dove, 2011).

Bearing all of this in mind, one can easily conclude that even abstract concepts and words are grounded. They might be dis-embodied (as in Dove, 2009; Dove, 2011), or exhibit various structural complexities (metaphorical, as in Lakoff \& Johnson, 1980; Lakoff \& Johnson, 1999; emotional, as in Kousta, Vigliocco et al., 2011; linguistic, as in Gleitman et al., 2005 or Vigliocco et al., 2004; relational, as in Wiemer-Hastings \& Xu, 2005 and Barsalou \& Wiemer-Hastings, 2005). However, authors do not discard the embodiment of abstract concepts and words, and Wiemer-Hastings \& Xu (2005) demonstrated that, surprisingly, some abstract concepts attach entity properties as well.

Since abstract words and concepts are more variable, i.e., associated with a wider variety of events, entities and situations, we hypothesize that many of them can be directly associated with, at least, some sensory domains. This may also be a probable cause for some of these to have entity-like properties (see Wiemer-Hastings \& Xu, 2005 and Barsalou \& Wiemer-Hastings, 2005). The hypothesis is not against listed views and findings, but merely an addition. Nevertheless, it contrasts with the dis-embodied view of Dove $(2009 ; 2011)$, which claims that a word's embodiment is arbitrarily related to its semantics.

We see the possibility that for many words and, furthermore, concepts, direct sensorimotor grounding can be an important part and a meaning carrier. As in LASS (Barsalou, Santos, Simmons, $\&$ Wilson, 2008), words and concepts are situated (c.f., Barsalou, 2005; Barsalou, 2009), and we think 
that their dis-embodiment (or arbitrariness) and embodiment can be a matter of degree, and are not necessarily mutually exclusive.

We believe that our claims would be suitably tested using a version of the methodology first introduced by Osgood. Nevertheless, we noticed that Barsalou, whose work inspired us, did not relate his work to Osgood's attempts and we had to ask why. There may be at least two reasons. First, Barsalou did propose a general theory of knowledge, while Osgood's research program aimed at understanding lexical semantic space. We agree that his general goal was indeed to understand cognitive general principles, but that it remained vastly overshadowed by his methodology. Second, Barsalou insisted on the prescription of sensory bases of all knowledge, while Osgood appeared to be seduced by the factor analytic approach (which was, at the time, state-ofthe-art), and this led him towards the description of the structure of semantic space. This again, could lead to the third point of divergence between Osgood and Barsalou; while the former ended in a reductionism by using factor analysis and pursuing minimal possible complexity (or dimensionality), the latter always acknowledged the full complexity of embodied cognition.

In this study we would like to offer further evidence for a general theory of knowledge, rather than a research program. Therefore we needed to alter Osgood's semantic differential paradigm. Additionally, in relation to his research program, we might say that the Osgood's three conclusions (Osgood, 1952, p. 226-227) were rather bold claims given the nature of the material used in his experiments. All of the material presented to participants was abstract: everything was labeled with words, from bipolar dimensions to the evaluated concepts. And those words could refer either to perceptual or conceptual domains. In other words, the material was an uncontrolled mixture of abstract and concrete conceptualizations. Finally, to the best of our knowledge, there was no control of the presented material, either of words and concepts, in terms of familiarity, frequency, complexity, similarity and so on. All of these methodological issues were carefully treated in the present study.

\section{Methodological issues: maps of meaning}

The way that data are formally modeled bears crucial significance to the subsequent theoretical interpretation. By pursuing a factor analytic approach, Osgood and his associates (1955; 1957; 1975) tried to determine an optimal number of abstract dimensions or continua. A factor analytic approach was also favored as it was presumed that the scales were highly intercorrelated (Osgood \& Luria, 1954). A three-factor solution was typically obtained (c.f., Jenkins, Russel, \& Suci, 1958; Osgood \& Suci, 1955), with the most salient factors of evaluation (good - bad), activity (active - passive), and potency (strong - weak). Nevertheless, according to Arnold (1971), there were two important drawbacks in Osgood's statistical enterprise. First, there is an indefinite number of factor solutions, even if one treats all orthogonal (or 'orthonormal', in Arnold's words) solutions as virtually the same. Second, semantic differential factors are not easily, if at all, translatable into dimensions that span distances of the semantic space. Distances, however, are natural means of inferring similarities and/or dissimilarities between concepts.

Methodologically speaking, within a specific experimental task, there is a clear difference between directly comparing the similarity (or dissimilarity) of two words, and finding a position for a word on a dimension spanned by some perceptual antonyms. The first approach is limited to abstract dyads of words, while the second approach alone is able to address the -possible space arrangements of concepts. Arnold (1971) proposed to disentangle the two types of comparisons through statistical tools, and applied methodology and statistics very different from those of Osgood (Arnold, 1971). He selected three sets of words (nouns, adjectives, and verbs), and asked participants to rate, pairwise, their similarities (or dissimilarities) in meanings. Then, the similarity rates obtained were multidimensionally scaled (MDS).

In parallel, the same three sets of words were rated on the three semantic differential factors: evaluation, activity and potency. Finally, Arnold (1971) had regressed three semantic differential 
factors with the MDS position-values. Although noun and adjective similarities predicted semantic differential dimensions quite well, the MDS dimensions and the semantic differential factors were not co-linear. In terms of applied statistics, one can easily note that distances (a spatial nearness) and correlations (a deviation similarity from an average) are rather different indicators.

Arnold's statistical approach in modeling semantic space might appear quite perplexing, since he was trying to ascertain the correlation between the distances (nearness) and the correlations (similarity). However, his remark about Osgood's factor analytic accomplishment remains valid, and his attempt to describe semantic space using distances is sound. Therefore we also used distances to define the semantic space.

Osgood (1952) claimed that words' semantic space is limited by our sensory nervous system. Despite this, in his research, he always used a mixture of abstract and concrete adjectives to create dimensions. Our choice of dimension was different from what is classically used as semantic differential since all of our dimensions came directly from perceptual domain. This worked well with the general attempt to contribute to knowledge about grounded cognition.

Finally, following Arnold (1971), we sought to establish whether our understanding of the semantic space could be facilitated when presented as a visual plane. Therefore we also tested our assumptions using two-dimensional response space, in an attempt to establish if it would mirror semantic spatiality more directly.

\section{Problem}

In this study we investigate whether Osgood and Barsalou are speaking about the same space, in fact - perceptual, limited by the sensory experiences and, ultimately, limited by the functioning of the nervous system. From our previous discussion, it seems obvious that Osgood's research program should fit into Barsalou's general theory of knowledge. Physical space, in which we live and gain experiences, is interfaced through our senses with semantic space. Thus, we can pose a question as to whether the later is isomorphous to the former. And, furthermore, is there a correspondence in dimensionality, from physical to semantic space.

We would also like to attend to the issue of abstract concepts and words that label them. As stated previously, we believe that all concepts are situated, and that for many abstract concepts, some sensory modalities, at least, can be an important part of their semantics.

Present research starts from methodological adjustments of Osgood's ideas, but aims to ask more general questions about semantics. For example, is it possible to establish whether meanings of concepts create a space? If so, is that space somehow similar to physical space - the only space we know about?

\section{Method}

Participants Fifty-seven undergraduate students of Psychology at the University of Novi Sad participated in the study, in exchange for partial course credits. All of them were fluent speakers of Serbian, with normal or corrected-to-normal vision.

Stimuli Five concrete and five abstract nouns were selected from the Frequency Dictionary of Contemporary Serbian Language (Kostić, 1999). All of them were regular feminine Serbian nouns, matched for the number of characters ( 5 to 6 characters; on average: 5.4 for concrete, and 5.6 for abstract nouns), and the stem frequency $(40<f<$ 70; on average: 56.2 for concrete, and 50.4 for abstract nouns; with equal variance: $F(4,4)=1.827$, $p=0.574$; and insignificant difference between means: $t(8)=0.905, p=0.394)$. Two groups of nouns differed significantly in average concreteness rate, as measured on 7-degree scale, from abstract to concrete (on average: 5.9 for concrete, and 2.39 for abstract nouns; with equal variance: $F(4,4)=$ $6.102, p=0.108$; and significant difference between means: $t(8)=20.030, p<0.001)$.

Unrelated linguistic items, divided into equal numbers of concrete and abstract words, were used to control for experiment-based artifacts (see discussion in Vigliocco et al., 2004). Furthermore, this control assured better scattering in resulting maps 
and, consequently, greater challenge in comparing obtained visual solutions. The small item set might be considered as the main disadvantage of the present research. It was however a conscious choice designed to keep the experimental session reasonably long. Still, we have found this to be a limiting factor for the generalizability of current findings, which is why our conclusions remained restrained.

\section{Scales}

Seven scales corresponding to three different sensory domains were constructed. Six of them were bi-polar: haptic (rough - smooth, wet-dry), auditory (loud - quiet, high pitch - low pitch), and visual (big - small, rectangular - rounded). The last scale also came from the visual domain but was not designed as bi-polar. It was a color array presented as a sequence of altering words or colors (purple, blue, red, green, yellow, orange, brown), along the dimension line. Thus, we maintained several original scales from Osgood (1952), disregarded some, which were more abstract (e.g., good - bad, or week - strong etc.), and adjoin a few of our own. Also we added one that was not bi-polar in the strict sense (color array). Principal restriction in constructing these scales was to remain as close to sensory domains as possible.

\section{Design and procedure}

The two experiments were scheduled six months apart, for the same sample of participants (repeated measurements). For Experiment 1 we simply replicated the original Osgood procedure (Osgood, 1952; Osgood, Suci, \& Tannenbaum, 1957; Osgood, May, \& Miron, 1975), using paper-and-pencil administering. Participants rated ten word items on seven semantic differential scales. A word was printed centrally on the paper sheet, followed by seven scales, one beneath the other. Then next word was printed, with its seven scales, and so on. An example of one experimental item is given in Figure 1.

For Experiment 2, we significantly modified the original design. First, we used computerized administering. Second, instead of one-dimensional scales, horizontally oriented, we used two-dimensional horizontal-vertical orientation: each of the seven scales appeared both horizontally (X-axis) and vertically oriented (Y-axis), and each of its orientation was combined with the remaining six scales. The overall set consisted of 84 two-dimensional combinations of original seven scales (7 scales combined with the remaining 6 , additionally rotated by 90 degrees: $7 \times 6 \times 2=84)$. The two-dimensional co-

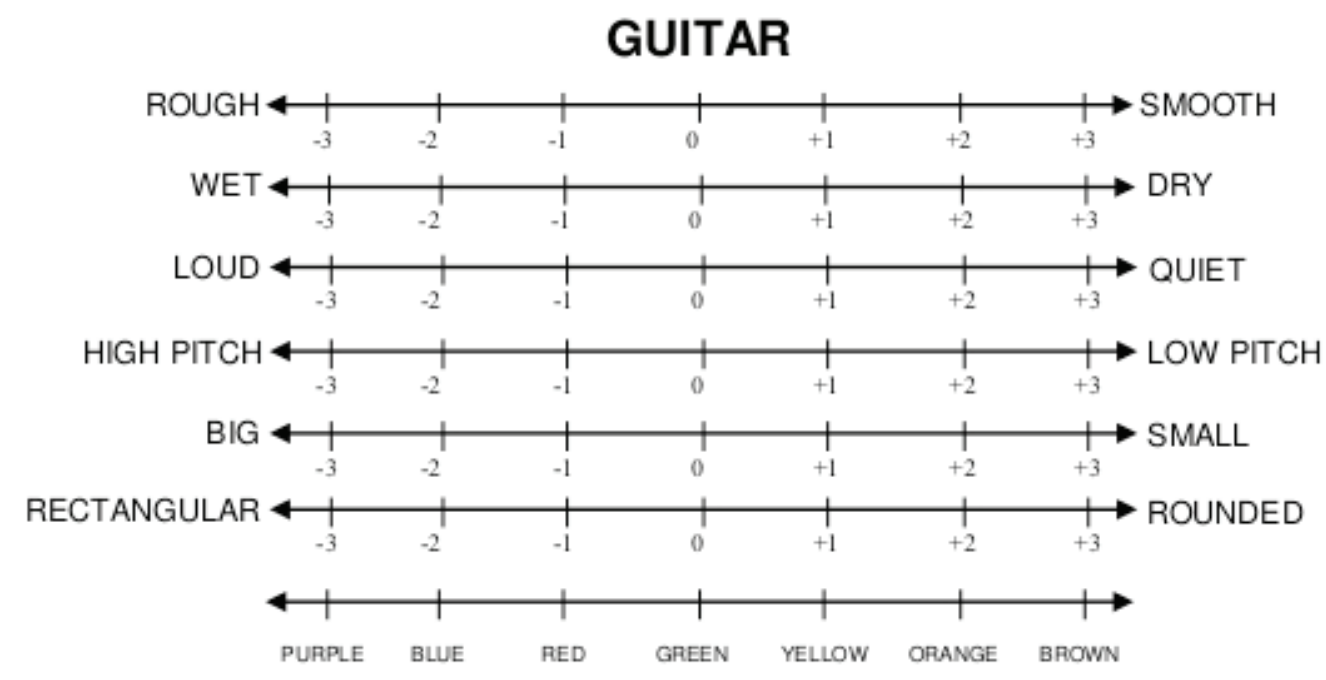

Figure 1. Example of a word item and seven semantic scales as they appeared in the paper-and-pencil form. Source: Own works.. 
ordinate system appeared centrally on the computer screen. A word item was marked with the "+" sign, on its left - at the word's beginning. A participant's task was to position the mouse on the " + " sign, then press the left mouse button, and to move a word item into what she/he considered to be a correct position on the two-dimensional semantic map (defined by a combination of the scales). Word items appeared one at the time on the computer screen. After a participant completed a task, she/he pressed the "NEXT" button to continue until completion. An example of the task is presented in Figure 2.

To control for the length of an experimental session, the whole list of the combination of two-dimensional maps (84) was divided into six balanced sub-lists, consisting of 14 maps. Participants were randomly assigned to one of the lists. Their task was to map all ten-word items on 14 ascribed maps. The ordering of both maps and word items was randomized per participant.
The procedure for the two experiments was standardized to the greatest extent possible. Except for the administration (paper-and-pencil vs. computer-assisted), Experiments 1 and 2 consisted of similar general instructions, examples, and several practice trials. Participants worked in groups of 20, at the beginning of regular course classes. Since there were three regular terms for a given course, each participant was allowed to enroll for the term at her/ his convenience. For Experiment 2 a computer-room was used, equipped with 25 standard IBM-compatible PCs, with the same configuration. Experimental sessions lasted approximately 20 minutes each.

Across two scheduled experiments, we systematically varied the scale orientation and the administration, ending with the three-level factor: traditional-horizontal (1DX), computerized-horizontal (2DX), and computerized-vertical (2DY). Dependent variables were rates, from Experiment 1, and positions on $\mathrm{X}$ and $\mathrm{Y}$-axes, from Experiment 2 .

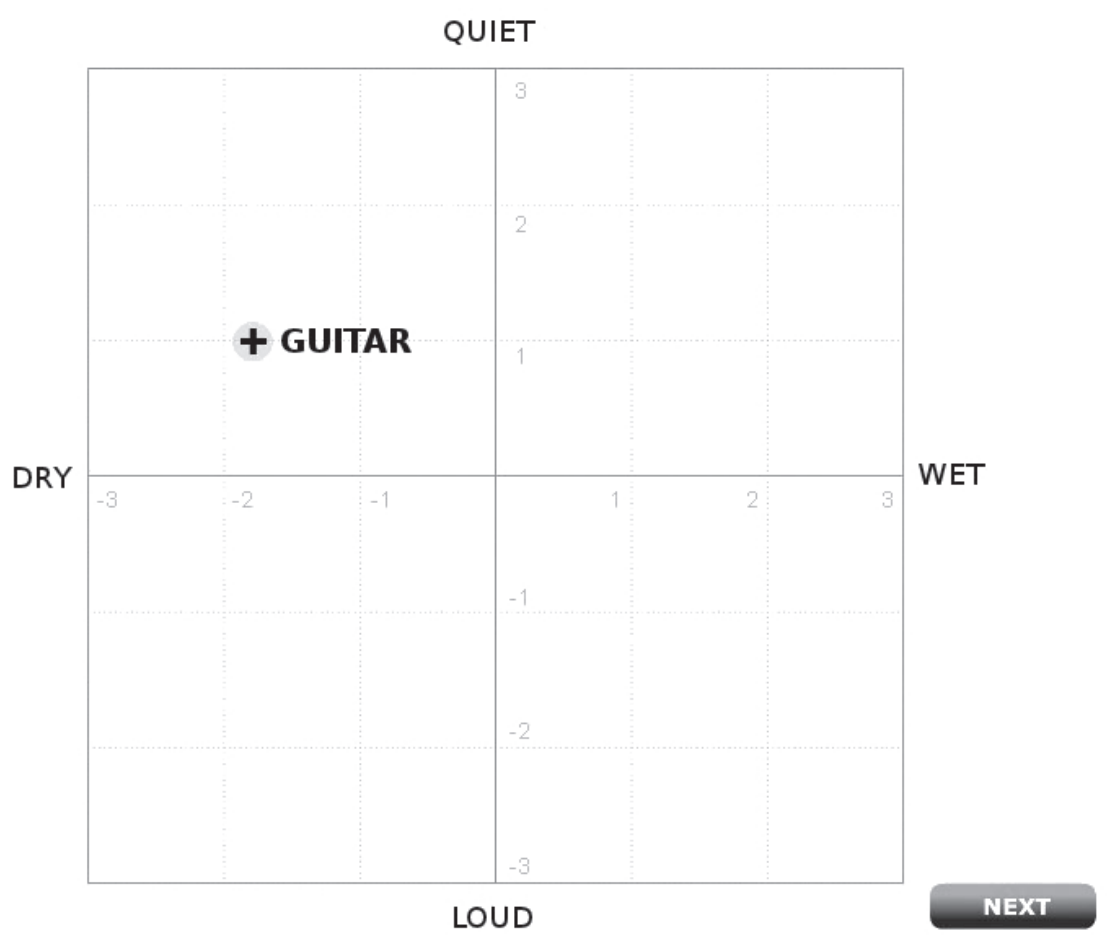

Figure 2. Example of a word item and two-dimensional combination of semantic scales as appeared in the computer application. Original application has less sharp contrast, using light gray for the background (here white), and dark gray for the foreground objects (here black).

Source: Own works. 


\section{Results}

A full dataset consisted of 11970 observations and 7 variables. A small number of observations containing missing rates were excluded from further analysis (0.3\%).

The first step in data analysis was to combine axis orientation (levels: $\mathrm{X}$-axis and Y-axis) and administration (levels: paper-and-pencil and computer-assisted) into experimental control factor with three levels: 1DX, 2DX and 2DY. This was convenient since the two original factors were nested, thus, having Y-axis in the computer-assisted experiment only. Furthermore, it allowed us to test both main effects of axis orientation and administration, and their interaction.

The analysis of by-participant average ratings showed significantly lower rates on $\mathrm{X}$-axis on two-dimensional maps, as compared with the traditional (paper-and-pencil) one-dimensional rates, and with Y-axis on the two-dimensional maps: F1 $(2,112)=5.296 ; p=0.006 ;$ contrasts using Tukey's test: $\mathrm{p}_{1 \mathrm{DX}-2 \mathrm{DX}}=0.006, \mathrm{p}_{1 \mathrm{DX}-2 \mathrm{DY}}=0.686, \mathrm{p}_{2 \mathrm{DX}-2 \mathrm{DY}}$ $=0.058$. At the same time, the by-item analysis showed that all differences were non-significant.

Since both analyses on average rates did not provide a consistent pattern of results, we applied mixed-effect modeling to test for the differences at the level of individual rates. Tests showed significant random-effects of participants, items and scales. In addition, there was a complex structure of random-effects, both with by-item and by-participant adjustments for the levels of our main factor the experimental control. Figure 3, which represents random-effects structure, reveals two interesting facts: word items vary more, while participants vary less in the case of traditional, one-dimensional paper-and-pencil administration (compare D1X vs. D2X and D2Y on the left and right panel in Figure 1). This finding is in line with the inconsistency between analyses on by-participant and by-item average rates.

From one point of view, the results could mean that traditional administration ensures slightly better discriminability of items, i.e., a better differentiation. At the same time, rates are somewhat more consistent or reliable. Thus, conclusions and recommendations must be in favor of traditional paper-and-pencil administration, and one-dimensional rating task.

Results could also mean that the two-dimension mapping task was, in principle, more challenging for participants. In particular, they showed less interpersonal agreement in positioning the word items on the $\mathrm{X}$-axis in the two-dimension mapping task (see lower left graph on the right-hand panel in Figure 3, which shows somewhat greater participants' variability). This also emerged as a significant contrast difference, between values on $\mathrm{X}$-axis and $\mathrm{Y}$-axis in the two-dimensional task (Wald's test: $\mathrm{p}_{2 \mathrm{DX}-2 \mathrm{DY}}=0.0246$ ). At the same time, the two other contrasts did not reach statistical significance (Wald's test: $\mathrm{p}_{1 \mathrm{DX}-2 \mathrm{DX}}=0.2258$, $\mathrm{p}_{1 \mathrm{DX}-2 \mathrm{DY}}$ $=0.3746$ ). Closer inquiry into differences revealed that they are not present across all seven scales, but only when "rough - smooth", "big - small" and "wet - dry" bi-polar scales are used. Also, on the "rectangular - rounded" scale there is a marginal difference. Overall, there is a relatively weak and unsystematic tendency in participants to give lower positioning of word item on the $\mathrm{X}$-axis in the two-dimensional mapping task. And this could be interpreted as the task bias.

However, the present constellation of results could also mean that participants' behavior was adjusted to the task, which requires the use of two dimensions, where finer or smaller changes on the map would be just good enough to match coarser and larger changes on the one-dimensional rating scale. In support of this view we can make use of the same findings as previously discussed: the lowering on the $\mathrm{X}$-axis in the two-dimensional mapping task was weak and unsystematic. In particular, although color scale was a major methodological deviation from original semantic differential, losing polarity or opposites and altering words (for the paper-and-pencil Experiment 1) and colors (for the computer-aided Experiment 2), the differences between conditions on this particular scale were non-significant, almost zero. Hence, from this too, we can infer consistency and robustness in participants' responses. 

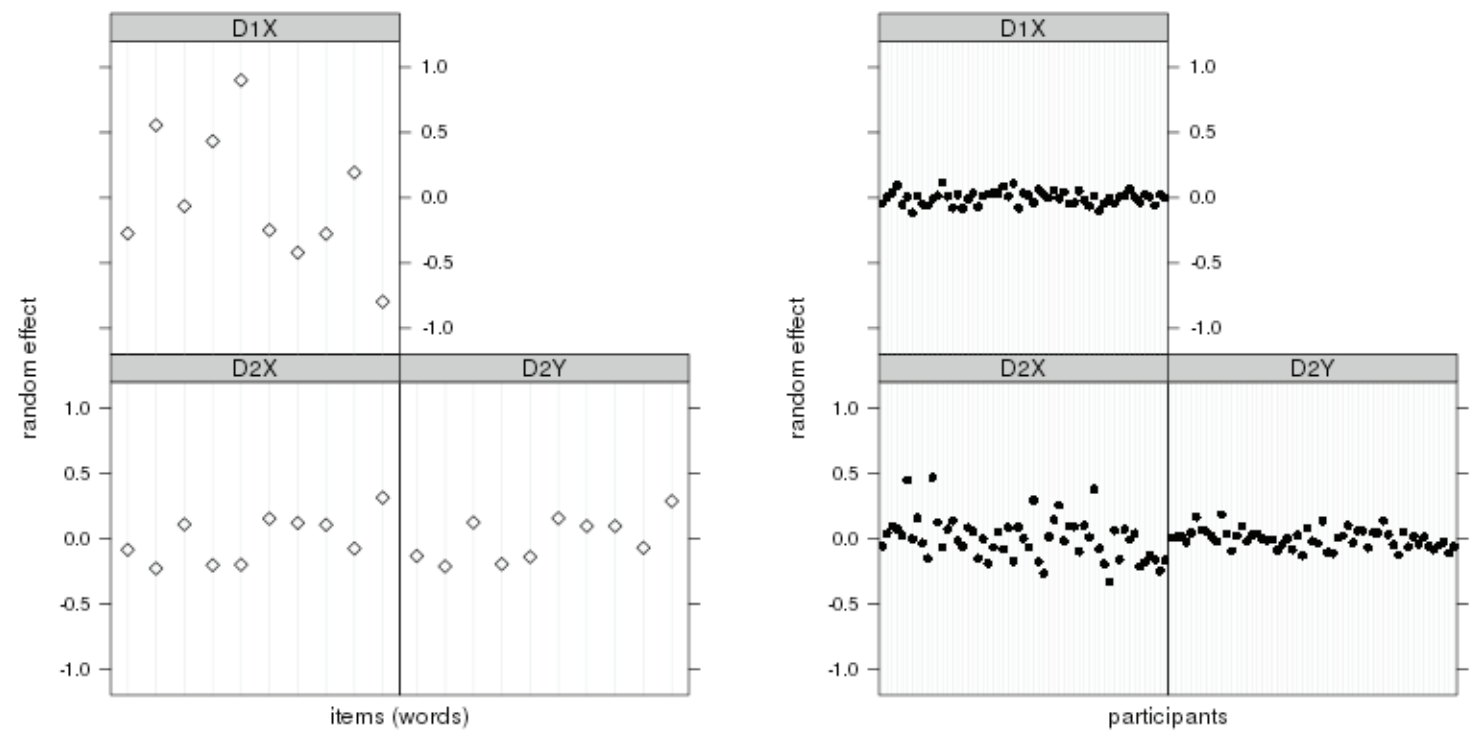

Figure 3. Adjustments for the experimental control factor to random-effects of items (left-hand panel) and participants (right-hand panel). D1X stands for one-dimensional horizontal (X-axis), D2X for two-dimensional horizontal (X-axis), and D2Y for two-dimensional vertical (Y-axis).

Source: Own works.

\section{The match between MDS and raw participants' mapping}

Repeated participants' measurements from the two experiments (traditional scaling vs. computer-aided mapping) provided tempting opportunity to test how well raw participants' two-dimensional maps fit to a statistical solution given by MDS from one-dimensional ratings. Crucially, MDS works under the assumption of minimal loss of information. Also, it typically represents structural relationships in terms of distances. Hence, if we can find that some, but not all of the raw two-dimensional maps are highly similar to the statistical, two-dimensional solution from MDS, then we are in position to conclude that this particular raw two-dimensional maps are both the most informative and distance based.

Before we ran the main analysis, we tested whether there is a significant difference in dispersion of word rates and/or mappings across seven scales. This is very important since MDS would be obsolete if words were scattered only on one or two dimensions, and "clumped" together on all remaining dimensions. There would be no information loss in discarding all and only uninformative (or non-discriminative) dimensions. In other words, a meaningful reduction in the number of dimensions needs comparable variability of items across all raw dimensions by applying MDS analysis.

Results showed overall non-significant difference in variability across scales, with only one marginally significant contrast between color scale and big - small scale (Tukey's test: Pcolors-bigsmall $=0.065)$. This reassured us to proceed with comparing raw two-dimensional maps with a statistical solution obtained by applying metric MDS.

Since scales used in experiments had standardized (or equidistant) units, we applied Classical (metric) MDS (see Gower, 1966; Cox \& Cox, 2001) on one-dimensional ratings from Experiment 1 . The statistical two-dimensional solution from MDS was then tested for similarity against all raw two-dimensional maps, i.e., by-participant average mappings from Experiment 2. We applied Monte Carlo test of a Procrustean rotations with 10000 permutation runs (for Monte Carlo test see Dray \& Dufour, 2007; and about Procrustean rotations and bootstrapping see Jackson, 1995; Chan et al., 1999; Peres-Neto \& Jackson, 2001). Results of the test revealed considerable differences in similarities, as represented in 
TABLE 1.

The strongest and the weakest average correlations between the two-dimensional solutions on ratings and from averaged raw mappings, both from MDS. Values are obtained with Monte Carlo Procrustean comparisons in 10000 permutation runs.

\begin{tabular}{cccc}
\hline X-axis & Y-axis & MC Procrustean rotations & p-value \\
\hline rectangular - round & rough - smooth & 0.933 & $>0.0001$ \\
rough - smooth & rectangular - round & 0.898 & $>0.0001$ \\
rough - smooth & loud - quiet & 0.833 & 0.0003 \\
$\ldots$ & $\ldots$ & $\ldots$ & $\ldots$ \\
wet - dry & loud - quiet & 0.358 & 0.5080 \\
wet - dry & high tone - low tone & 0.333 & 0.5750 \\
high tone - low tone & wet - dry & 0.316 & 0.5947 \\
\hline
\end{tabular}

Source: Own works.

Figure 4. Six raw two-dimensional maps having the highest and the lowest similarities with the statistical solution are also summarized in Table 1.

Table 1 reveals that the first two maps with the highest similarity represent the same combination of opposites, simply rotated for 90 degrees. Two-dimensional maps that combine rectangular - round and rough - smooth poles are the most informative. They exceed other combinations considerably. Again, the least informative maps combine high tone - low tone and wet - dry opposites. We believe that this order of maps is specific to a particular set of word items, and is not universal across a universe of possibilities.

The similarity between two solutions - the "statistical" and the "cognitive" - is not only captured by the Monte Carlo Procrustean test, but is visually obvious as well. The most similar maps, as given by the Monte Carlo Procrustean test, are presented in Figure 5.

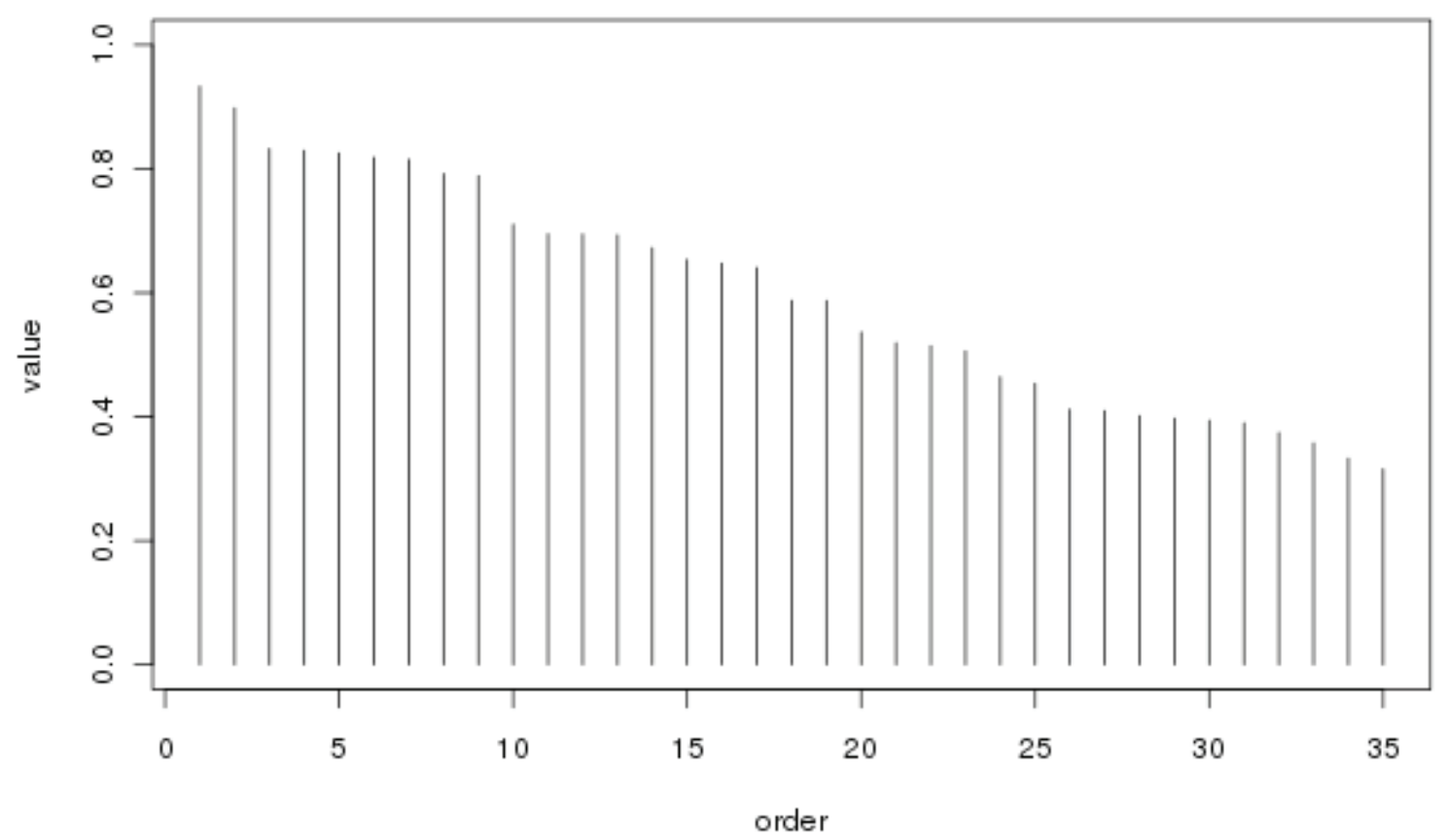

Figure 4. Average correlations between the two-dimensional solutions, from MDS on ratings, and from averaged raw mappings. Values are obtained with the Monte Carlo Procrustean comparisons in 10000 permutation runs. Source: Own works. 


\section{Can sensory opposites capture concreteness?}

To test our hypothesis that sensory modalities can be an important part of semantics even for abstract words, we tested whether the dimensions attested for their informativeness can be used to discriminate between concrete and abstract words that formed our stimulus set. Recall that the set consisted of five concrete and five abstract words, which differed significantly in their concreteness. Hence, we restrained ourselves from making generalizations based on such a small set of words. Rather, we remained within the nearest proximity of empirical findings, to observe if the dimensions, isolated by applying MDS, made accurate back-classification of words into concrete and abstract ones.

We applied linear logistic regression, which showed that the first MDS-dimension predicts concreteness (Wald's test: $z=6.147, \mathrm{p}<0.001$ ), where abstract items tend to get lower values on that dimension, showing to be less "smooth" than the concrete words. Since we established a similarity between that MDS-dimension and the rough smooth scale (consult Table 1 and Figure 5), we ran separate analyses that used raw $\mathrm{X}$-axis and Y-axis values obtained from the computer-assisted two-dimensional mapping, to reconfirm the above findings. Results on both axes showed that the very same scale, namely rough - smooth, was indeed the most predictive of concreteness (Wald's test for X-axis: $z=5.163, \mathrm{p}<0.001$; Wald's test for Y-axis: $z=4.693, p<0.001)$. Additionally, some other raw $\mathrm{X}$ and $\mathrm{Y}$ positions predicted concreteness as well, but to a lesser degree (in decreasing order: big - small, rectangular - rounded, high pitch - low pitch; color array was predictive if on $\mathrm{X}$-axis, and wet - dry was predictive if on Y-axis). The second MDS-scale and the remaining raw $\mathrm{X}$ and $\mathrm{Y}$ positions on the two-dimensional maps were found to be non-discriminative of words' concreteness.

\section{Discussion}

The intention of this study was to model the representational space of concepts. We were motivated by the idea that sensory experience shapes our cognition (Barsalou, 1999; Barsalou, 2008; Gibbs, 2006). In order to gather the data to model such
1D (paper-and-pencil)

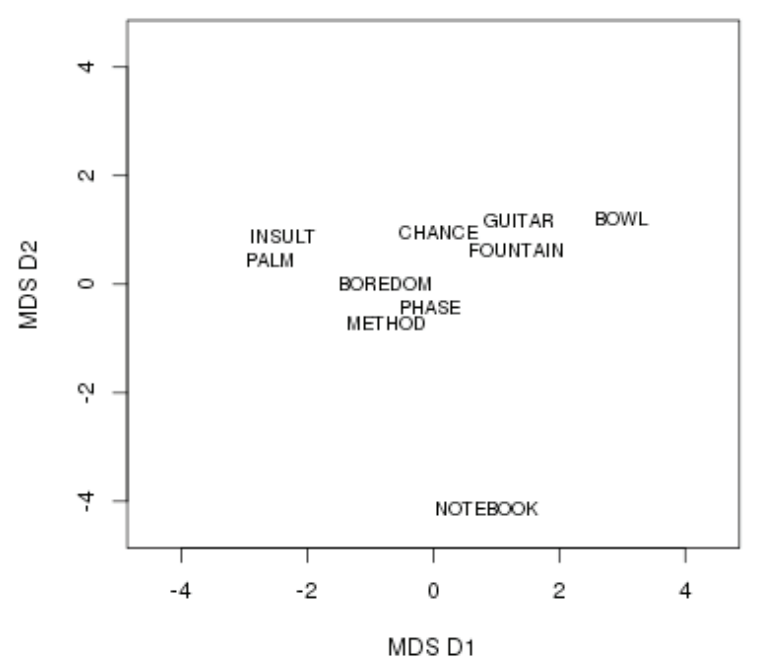

2D (computer-based)

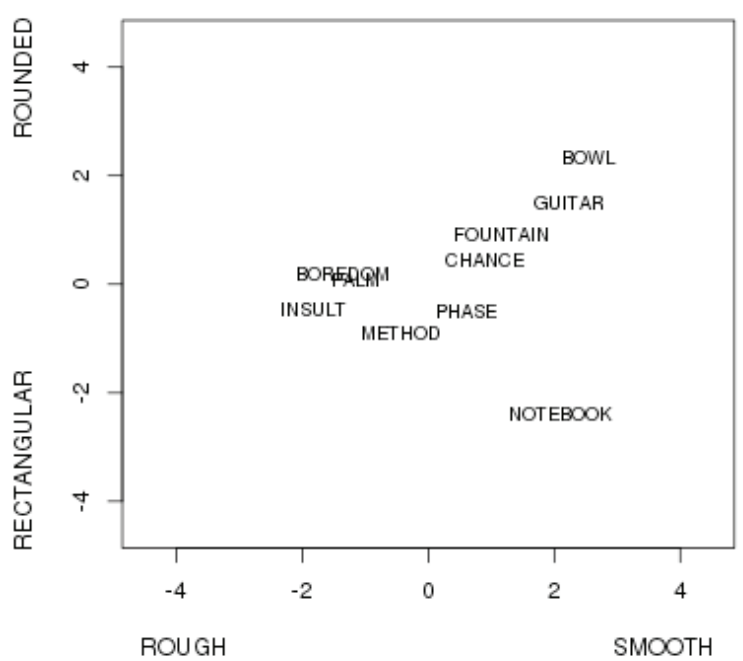

Figure 5. Two-dimensional maps representing scattering of word items in semantic space(s). The left-hand side map shows statistical solution from the MDS using traditional, paper-and-pencil ratings. The right-hand side map is obtained directly from by-participant average mappings, in computer-aided task.

Source: Own works. 
mental space we used semantic differential ratings, originally developed by Osgood and his associates (Osgood, 1952; Osgood, Suci, \& Tannenbaum, 1957; Osgood, May, \& Miron, 1975). We then modified Osgood's methodology by asking our participants to construct semantic maps directly, in the two-dimensional plane, which imposed spatiality in semantic differentials. Finally, MDS was applied to the data from the traditional rating task, obtaining a two dimensional solution - a map - comparable with the data from the novel mapping task.

From a statistical point of view, bi-dimensional mapping exhibits differences from traditional, uni-dimensional rating. In our opinion, this difference is due to participants' adjustment to the task: as they had two-dimensions at their disposal, they made use of them, relying less on one dimension only but engaging the second as well. Alternative explanations were inspired by the fact that only the horizontal or X-axis in the two-dimensional mapping task differed, both from the vertical or Y-axis in the same task and from the one-dimensional rating task (also horizontally oriented). These seem more cumbersome to us. This is especially the case considering that those differences were relatively mild and that they occurred only on three out of seven scales.

The fact that we did not find any statistical differences between the paper-and-pencil and computer-aided administration, nor between the verbal and non-verbal color scale across the two experiments, also aligns with our hypothesis that participants' behavior was optimal, consistent and robust at the same time. However, we would like to underline that verbal - non-verbal sameness should not be generalized from the one and only case of color scale. It is not even a bi-polar scale, which could only add to its idiosyncratic status in the present research and in general. However, the identification of continua, which could be easily represented perceptually without the actual use of symbols, is not easy; and many of those symbols would simply be poor replacements for abstract poles of continua. For example, in order to specify a rectangular round continuum, it is not adequate to choose one random representative of the class, for example square and circle. Similarly, being shown a glass of water and a piece of cloth does not necessarily represent wet - dry opposites.

What found to be common, on the other hand, is a high similarity between the statistical two-dimensional solution, obtained from MDS, and raw by-participant average mappings in the rectangular - round and rough - smooth plane. By default, the loss of information in MDS is minimal; thus, we conclude that the raw map spanning rectangular - round and rough - smooth is highly informative. At the same time, similarity provides evidence that the map represents distances.

It did not come as a surprise that a scale from the visual domain is informative, or important. Although this could also be due to the visual presentation of material, we identify that the second bi-polar scale (rough - smooth) spanned opposites from the haptic domain and that the same scale occurred in all three most informative maps. In any circumstances, cross-modal information profusion is consistent with more recent tendencies in the field of perception (Ghazanfar \& Schroeder, 2006; Shimojo \& Shams, 2001). They emphasize multi-modality of our experience, where studying each sense separately only distorts our understanding. Our phenomenology confirms these ideas; it is difficult to think of an actual object that is experienced through a single sense.

For the purpose of this initial study, we have chosen several scales of particular interest. In fact we believe that they may be important for our conscious experience of the world. It would be useful to test other scales based on the same modalities, but representing less frequent and/or less prominent dimensions, like transparent-opaque for example. It would also be useful to test more than these three modalities, especially those we do not use frequently to describe objects, scenes or events. The importance of the certain scale must be heavily influenced by the particular concepts used in the study. For example, words denoting food or drink would heavily rely upon chemical or thermal senses. We would advocate, then, many new words, and additional modalities described with appropriate adjectival opposites, to be used in future studies. 
As a second step, we would suggest the introduction of adjectival opposites that could capture the complexity of semantics to its fullest richness. Thus, one should follow the empirical findings from previous studies and include, for example, emotional states (Kousta, Vigliocco et al., 2011), introspective states (Barsalou, 2005), temporal and other relations (Barsalou \& Wiemer-Hastings, 2005), and many other possible opposites (also consult: Gleitman et al., 2005; Vigliocco et al., 2004; Lakoff \& Johnson, 1999; Johnson \& Lakoff, 2002). This would ensure anti-reductionism, which is typical for most of the grounded cognition frameworks.

At the same time, we do not wish to over-generalize the present findings. The small set of sensory opposites that we used in the current research did not capture the full richness of the semantics of word items. Yet particular dimensions were highly predictive of concreteness, which is a very important and pervasive feature of meaning. Results showed that abstract items are rated and mapped as less "smooth". Interestingly, however, this smoothlessness of abstract word items can be metaphorical (c.f., Lakoff \& Johnson, 1980; Lakoff \& Johnson, 1999; Gibbs, 1994; Gibbs, 2006), or can be understood as a lexical (or linguistic) interface (c.f., Gleitman et al., 2005; Vigliocco et al., 2004) to the emotional states (c.f., Kousta, Vigliocco et al., 2011) and other introspective states (c.f., Barsalou, 2005; Barsalou, 2009). This could even mean that they are more complex and variable, and, hence, less "smooth". Nevertheless, present empirical findings are insufficient to provide a definite answer as to which of the enlisted interpretations is the right one. They only provide a strong case against the claim that the concepts are dis-embodied, only arbitrarily related to their semantics (c.f., Dove, 2009; Dove, 2011).

We found enough evidence to conclude that cognitive semantic space mirrors the space in which we live and gain experiences. Our senses do serve as mirrors, as the grounded cognition framework would predict (Barsalou, 1999; Barsalou, 2008; Gibbs, 2006). This semantic space of ours seems to respond to the physical space; it is shaped by information about the world that we get through our senses. Our findings should be related to other recent findings in the domain of semantic features that use a graded measure of semantic similarity (e.g., Cree \& McRae, 2003; Vigliocco et al., 2004). However, our aim and scope was less ambitious and general. Considering the small number of word items, in particular, this was more of an exploration, from one of Osgood's original claims, to grounded cognition frameworks, via the adaptation of Osgood's semantic differential methodology.

\section{Conclusion}

We have presented one possible model of semantic space. The abstract space we defined is created from the sense data resulting from a number of perceptual modalities. The space itself is a dynamic mental representation that varies depending on a particular sequence of sensory events during the lifespan. It is not hard to imagine the perceptually grounded mental representation of concrete concepts. However, we would agree with the grounded cognition camp, claiming that even abstract concepts must be embodied. As Barsalou (1999), and many before him, proposed, our knowledge is perceptual in nature. For Gleitman and her collaborators, the same is true for the meanings of words (Gleitman et al., 2005; in addition, for the language-to-world mapping of spatial perspective, see Lee \& Gleitman, 2002). This could explain why we seek tools from our senses to aid our understanding, especially in cases of complex and abstract phenomena.

The present findings support the multidimensionality of conceptual space, the perceptual basis of knowledge and the dynamic characteristics of the concepts discussed in contemporary theories.

\section{References}

Arnold, J. B. (1971). A Multidimensional Scaling Study of Semantic Distance. Journal of Experimental Psychology, 90, 349-372.

Barsalou, L. W. (1991). Deriving Categories to Achieve Goals. In G. H. Bower (Ed.), The psychology of learning and motivation: Advances in research and theory (Vol. 27, pp. 1-64). San Diego: Academic Press. 
Barsalou, L. W. (1992). Frames, concepts, and conceptual fields. In E. Kittay \& A. Lehrer (Eds.), Frames, fields, and contrasts: New essays in lexical and semantic organization (pp. 21-74). Hillsdale: Lawrence Erlbaum.

Barsalou, L. W. (1993). Flexibility, structure, and linguistic vagary in concepts: Manifestations of a compositional system of perceptual symbols. In A. C. Collins, S. E. Gathercole, \& M. A. Conway (Eds.), Theories of memory (pp. 29-101). London: Lawrence Erlbaum.

Barsalou, L. W. (1999). Perceptual symbol systems. Behavioral and Brain Sciences, 22, 577-609.

Barsalou, L. W. (2005). Situated conceptualization. In H. Cohen, \& C. Lefebvre (Eds.), Handbook of categorization in cognitive science (pp. 619-650). St. Louis: Elsevier.

Barsalou, L. W. (2008). Grounded cognition. Annual Review of Psychology, 59, 617-645.

Barsalou, L. W. (2009). Simulation, situated conceptualization, and prediction. Philosophical Transactions of the Royal Society of London: Biological Sciences, 364, 1281-1289.

Barsalou, L. W., Santos, A., Simmons, W. K., \& Wilson, C. D. (2008). Language and simulation in conceptual processing. In M. De Vega, A. M. Glenberg, \& A. C. Graesser (Eds.), Symbols, Embodiment, and Meaning (pp. 245-284). Oxford: Oxford University Press.

Barsalou, L.W., \& Wiemer-Hastings, K. (2005). Situating abstract concepts. In D. Pecher, \& R. Zwaan (Eds.), Grounding cognition: The role of perception and action in memory, language, and thought (pp. 129-163). New York: Cambridge University Press.

Boroditsky, L., \& Ramscar, M. (2002). The roles of body and mind in abstract thought. Psychological Science, 13, 185-188.

Chan, W., Ho, R. M., Leung, K., Chan, D. K. S., \& Yung, Y. F. (1999). An alternative method for evaluating congruence coefficients with Procrustes rotation: A bootstrap procedure. Psychological Methods, 4, 378-402.

Cox, T. F., \& Cox, M. A. A. (2001). Multidimensional Scaling. Boca Raton: Chapman \& Hall/CRC.

Cree, G. S., \& McRae, K. (2003). Analyzing the factors underlying the structure and computation of the meaning of chipmunk, cherry, chisel, cheese, and cello (and many other such concrete nouns). Journal of Experimental Psychology: General, 132, 163-201.

Dove, G. O. (2009). Beyond perceptual symbols: a call for representational pluralism. Cognition, 110, 412-431.

Dove, G. O. (2011). On the need for embodied and dis-embodied cognition. Frontiers in Psychology, 1 , article 242.

Dray, S., \& Dufour, A. B. (2007). The ade4 package: implementing the duality diagram for ecologists. Journal of Statistical Software, 22, 1-20.

Ghazanfar, A. A., \& Schroeder, C. E. (2006). Is Neocortex essentially multisensory? Trends in Cognitive Sciences, 10, 278-285.

Gibbs, R. W. Jr. (1994). The Poetics of Mind: Figurative Thought, Language, and Understanding. New York: Cambridge University Press.

Gibbs, R. W. Jr. (2006). Embodiment and Cognitive Science. New York: Cambridge University Press.

Gleitman, L. R., Cassidy, K., Papafragou, A., Nappa, R., \& Trueswell, J. T. (2005). Hard words, Journal of Language Learning and Development, 1, 23-64.

Gower, J. C. (1966). Some distance properties of latent root and vector methods used in multivariate analysis. Biometrika, 53, 325-328.

Huettig, F., \& Altmann, G. (2011). Looking at anything that is green when hearing 'frog': How object surface colour and stored object colour knowledge influence language-mediated overt attention. Quarterly Journal of Experimental Psychology, 64, 122-145.

Jackson, D.A. (1995). PROTEST: A PROcrustean randomization TEST of community environment concordance. Ecosciences, 2, 297-303.

Jenkins, J. J., Russell, W. A., \& Suci, G. J. (1958). An Atlas of Semantic Profiles for 360 Words. The American Journal of Psychology, 71, 688-699.

Johnson, M., Lakoff, G. (2002). Why cognitive linguistics requires embodied realism. Cognitive Linguistics, 13, 245-263.

Kousta, S. T., Vigliocco, G., Vinson, D. P., Andrews, M., \& Del Campo, E. (2011). The representation of abstract words: Why emotion matters. Journal of Experimental Psychology: General, 140, 14-34. 
Lakoff, G., \& Johnson M. (1980). Metaphors We Live By. Chicago: University of Chicago Press.

Lakoff, G., \& Johnson M. (1999). Philosophy in the Flesh: The Embodied Mind and Its Challenge to Western Thought. New York: Basic Books.

Lee, P., \& Gleitman, L. (2002). Turning the tables: language and spatial reasoning. Cognition, 83, 265-294.

Lupyan, G. (2012). Linguistically modulated perception and cognition: the label feedback hypothesis. Frontiers in Cognition, 3, article 54.

Marr, D. (1982). Vision. San Francisco: Freeman.

Osgood, C. E. (1952). The Nature and Measurement of Meaning. Psychological Bulletin, 49, 197-237.

Osgood, C. E., \& Luria, Z. (1954) A blind analysis of a case of multiple personality using the se- mantic differential. Journal of Abnormal and Social Psychology. Vol 49: 579-91.

Osgood, C. E., \& Suci, G. J. (1955). Factor analysis of meaning. Journal of Experimental Psychology. Vol 50(5), 325-338.

Osgood, C. E., May, W. H., \& Miron, M. S. (1975). Cross-Cultural Universals of Affective Meaning. Urbana: University of Illinois Press.

Osgood, C. E., Suci, G. J., \& Tannenbaum, P. H. (1957). The Measurement of Meaning. Urbana: University of Illinois Press.

Paivio, A. (1986). Mental representations: A Dual-Coding Approach. New York: Oxford University Press.

Peres-Neto, P. R., \& Jackson, D. A. (2001). How well do multivariate data sets match? The advantages of a Procrustean superimposition approach over the Mantel test. Oecologia, 129, 169-178.
Sadoski, M., \& Paivio, A. (2004). A dual coding theoretical model of reading. In R. B. Ruddell, \& N. J. Unrau (Eds.) Theoretical models and processes of reading (pp. 1329-1362). Newark: International Reading Association.

Shimojo, S., \& Shams, L. (2001). Sensory modalities are not separate modalities: plasticity and interactions. Current Opinion in Neurobiology, 11, 505-509.

Spivey, M. J., Tyler, M. J., Eberhard, K. M., \& Tanenhaus, M. K. (2001). Linguistically Mediated Visual Search. Psychological Science, 12, 282-286.

Wolpert, D. M., Ghahramani, Z., \& Flanagan, J. R. (2001). Perspectives and problems in motor learning. Trends in Cognitive Science, 5, 487-494.

Vigliocco, G., Meteyard, L., Andrews, M., \& Kousta, S. (2009). Toward a theory of semantic representation. Language and Cognition, 1, 219-247.

Vigliocco, G., Vinson, D. P., Lewis, W., \& Garrett, M. F. (2004). Representing the meanings of object and action words: The featural and unitary semantic space hypothesis. Cognitive Psychology, 48, 422-488.

Wiemer-Hastings, K., \& Xu, X. (2003). Thematic integration in the similarity of abstract and concrete items. In Proceedings of the 25th Annual Meeting of the Cognitive Science Society, Boston. MA.

Wiemar-Hastings, K., \& Xu, X. (2005). Content differences for abstract and concrete concepts. Cognitive Science, 29, 719-736.

Yee, E., Ahmed, S.Z., \& Thompson-Schill, S. L. (2012). Colorless Green Ideas (Can) Prime Furiously. Psychological Science, 23, 364-369. 